\title{
University of Oxford
}

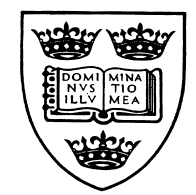

\section{Clinical Trial Protocol}

Study Reference: 006

A Phase I study of the safety and immunogenicity of BCG (Bacille Calmette-Guerin) vaccine delivered intradermally by a needle injection in healthy volunteers who have previously received BCG.

Version 2; 22.6.2004

\section{CONFIDENTIAL}




\section{INTRODUCTION AND BACKGROUND}

\subsection{The need for new vaccine against tuberculosis}

Tuberculosis (TB) kills about three million people annually. It is estimated that one third of the world's population are latently infected with Mycobacterium tuberculosis (M.tb). Multi-drug resistant strains of M.tb, and co-infection with M.tb and HIV present major new challenges. The currently available vaccine, $M$. bovis BCG, is largely ineffective at protecting against adult pulmonary disease in endemic areas and it is widely agreed that a new more effective tuberculosis vaccine is a major global public health priority ${ }^{1}$. However, it may be unethical and impractical to test and deploy a vaccine strategy that does not include BCG, as BCG does confer worthwhile protection against TB meningitis and leprosy. An immunisation strategy that includes BCG is also attractive because the populations in which this vaccine candidate will need to be tested will already have been immunised with BCG.

M.tb is an intracellular organism. CD4+ Th1-type cellular responses are essential for protection and there is increasing evidence from animal and human studies that CD8+ T cells also play a protective role ${ }^{2}$. However, it has generally been difficult to induce strong cellular immune responses in humans using subunit vaccines. DNA vaccines induce both CD4+ and CD8+ T cells and thus offer a potential new approach to a TB vaccine. DNA vaccines encoding various antigens from $\mathrm{M}$. tuberculosis have been evaluated in the murine model, and to date no DNA vaccine alone has been shown to be superior to $\mathrm{BCG}^{3,4}$.

A heterologous prime-boost immunisation strategy involves giving two different vaccines, each encoding the same antigen, several weeks apart. Such regimes are extremely effective at inducing a cellular immune response. Using a DNA- prime/MVA-boost immunisation strategy induces high levels of CD8+ T cells in animal models of malaria and HIV ${ }^{5,6}$, and high levels of both CD4+ and CD8+ T cells in animal models of TB ${ }^{7}$. BCG immunisation alone induces only CD4+ T cells in mice. A prime-boost strategy using BCG as the prime and a recombinant MVA encoding an antigen from M.tb that is also present in BCG (antigen 85A: 'MVA85A') as the boost, induces much higher levels of CD4+ T cells than BCG or MVA85A alone. In addition, this regime generates specific CD8+ T cells that are undetectable following immunisation with BCG alone.

\subsection{Recombinant viruses as vaccines.}

Recombinant viruses used alone have for some years represented a promising vaccine delivery system, particularly for inducing cellular immune responses ${ }^{8}$. The recombinant virus encodes the immunising protein or peptide. Immunisation by a recombinant virus vaccine occurs when host cells take up and express the inoculated attenuated virus encoding a protective antigen ${ }^{9}$. The expressed protein often has the native conformation, glycosylation, and other post-translational modifications that occur during natural infection. Recombinant viral vaccines may elicit both antibody and cytotoxic T-lymphocyte responses ${ }^{10}$, which persist without further immunisations. Many viruses have been investigated as potential recombinant vaccines. The successful worldwide eradication of smallpox via vaccination with live vaccinia virus highlighted vaccinia

as a candidate for recombinant use ${ }^{11,12,13}$. The recognition in recent years that non-replicating strains of poxvirus such as MVA and avipox vectors can be more immunogenic than traditional replicating vaccinia strains has enhanced the attractiveness of this approach. MVA (modified 
vaccinia virus Ankara) is a strain of vaccinia virus which has been passaged more than 570 times though avian cells, is replication incompetent in human cell lines and has a good safety record. It has been administered to more than 120,000 vaccinees as part of the smallpox eradication programme, with no adverse effects, despite the deliberate vaccination of high risk groups ${ }^{14,15}$. This safety in man is consistent with the avirulence of MVA in animal models ${ }^{16}$. MVA has six major genomic deletions compared to the parental genome severely compromising its ability to replicate in mammalian cells ${ }^{17}$. Viral replication is blocked late during infection of cells but importantly viral and recombinant protein synthesis is unimpaired even during this abortive infection ${ }^{18}$. Replication-deficient recombinant MVA has been seen as an exceptionally safe viral vector $^{19,20}$. When tested in animal model studies recombinant MVAs have been shown to be avirulent, yet protectively immunogenic as vaccines against viral diseases and cancer $\mathrm{r}^{6,21,22,23,24}$. The most useful data on the safety and efficacy of various doses of a recombinant MVA vaccine comes from clinical trial data with a recombinant MVA expressing a number of CTL epitopes from Plasmodium falciparum pre-erythrocytic antigens fused to a complete pre-erythrocytic stage antigen, Thrombospondin Related Adhesion Protein (TRAP). These trials have given a total of 169 immunisations with this recombinant MVA, to 49 UK vaccinees 38 Gambian vaccines (20 of whom were children aged 1-5). 6 doses of $1 \times 10^{7} \mathrm{pfu}, 139$ doses of $5 \times 10^{7} \mathrm{pfu}$, 6 doses of $1 \times 10^{8}$ pfu and 18 doses of $2.5 \times 10^{8}$ pfu have been administered, all without serious adverse effects.

\subsection{Recombinant MVA encoding antigen 85A}

Secreted antigens from M. tuberculosis are released from actively metabolising bacteria, and are important targets in protective immunity ${ }^{25}$. Antigen $85 \mathrm{~A}$ is a major secreted antigen from $M$. tuberculosis which forms part of the antigen 85 complex (A, B and C). This complex constitutes a major portion of the secreted proteins of both M.tb and BCG. It is involved in fibronectin binding within the cell wall and has mycolyltransferase activity ${ }^{26}$. MVA85A induces both a CD4+ and a CD8+ epitope when used to immunise mice. When mice are primed with BCG and then given MVA85A as a boost, the levels of CD4+ and CD8+ T cells induced are higher than with either BCG or MVA85A alone.

We are evaluating the safety and immunogenicity of the following 3 groups:

1. BCG alone

2. MVA85A alone

3. BCG prime-MVA85A boost

BCG-BCG provides a control group for BCG-MVA85A. Many countries have a tradition of repeated BCG vaccination and the criteria for revaccination differ between countries ${ }^{27}$.

\section{DESCRIPTION OF THE STUDY}

\subsection{Study Objective}

To assess the safety and immunogenicity of BCG delivered intradermally into the deltoid region in volunteers who have received BCG 10 - 20 years previously. 


\subsection{Selection of volunteers}

Volunteers for the study will be recruited through advertisements. Each volunteer will have received an information sheet concerning the study and will have agreed to participate in writing. Volunteers will be given at least 48 hours between reading the information leaflet and agreeing to participate. Female volunteers will be told of the theoretical risk of congenital anomaly should they become pregnant during the study and only those who undertake to take precautions to avoid pregnancy during the study period will be eligible. Volunteers will give signed consent for their GP's to be notified about their participation in the trial. The GP will be faxed a letter on the day of screening and asked to reply if they know of a reason why the volunteer should not take part. The signed consent form will also be faxed with the letter.

\subsection{Screening}

Volunteers will be asked to sign the informed consent form for screening. The following will be performed:

- Medical history and examination

- Laboratory evaluations - including clinical chemistry, haematology, HLA typing, antiHBV antibodies, anti-HCV antibodies, anti-HIV antibodies

- Heaf test - to exclude prior exposure to TB

- Urinalysis and urine pregnancy test if female

\subsection{Inclusion Criteria}

- Healthy adult aged 18-55 years.

- Normal medical history and physical examination.

- Normal urine dipstick, blood count, liver enzymes, and creatinine.

\subsection{Exclusion Criteria}

a. Exposure to TB at any point. A positive ESAT6/CFP10 Elispot response (defined as greater than 5 spots/well above background and at least double the background response).

b. Clinically significant history of skin disorder (eczema, psoriasis, etc.), allergy, immunodeficiency, cardiovascular disease, respiratory disease, endocrine disorder, liver disease, renal disease, gastrointestinal disease, neurological illness, psychiatric disorder, drug or alcohol abuse.

c. Oral or systemic steroid medication or the use of immunosuppressive agents.

d. Positive HIV antibody test, HCV antibody test or positive HBV serology except postvaccination.

e. Heaf test greater than Grade II

f. Confirmed pregnancy

\subsection{Withdrawal Criteria}

a. Withdrawal of consent by subject for any reason 
b. Loss to follow-up

c. Non-compliance with study procedures

d. Protocol violation

e. Serious adverse event (as defined in Appendix 3)

f. Any other reason at discretion of the Principal Investigator

g. Confirmed pregnancy during study period

\subsection{Immunisation}

On Day 0, subjects will receive a single intradermal injection of $0.1 \mathrm{ml}$ BCG over the deltoid muscle. Vital signs will be monitored at 30 and 60 minutes post-immunisation. Local reactions at the site of administration will be evaluated at 60 minutes.

A photograph of the injection site may be taken at 48 hours (with written consent). The injection site will be reviewed 7 days after each immunization.

Blood will be taken at the following time points: At the screening visit*, prior to the vaccination, 1 week after the first vaccination*, 2 weeks, 4 weeks, 8 weeks, 12 weeks* and 24 weeks after the vaccination. Up to $55 \mathrm{mls}$ will be taken at any one time with the total being no more than 500 mls over the study period. *Samples taken on these dates will be tested for full blood count and biochemical screen. Immunological assays will be performed at all time points to determine vaccine immunogenicity. A pregnancy test will be performed prior to vaccination for female volunteers. Peripheral blood mononuclear cells will be prepared for cellular immunological assays to be performed without or following cryopreservation. Other serological measures of immune response, i.e. antibody titres, will be assayed on frozen plasma samples.

All blood tests will be taken within 1-3 days of the due date as described in the schedule above.

At the 24 week timepoint, a Heaf test will be repeated in order to document the change (if any) in Heaf responsiveness after the second BCG vaccination.

\subsection{Endpoints}

The occurance and severity of local side-effects

The occurance and severity of systemic side-effects

The induction of $\mathrm{T}$ cell responses (as measured by an interferon-gamma Elispot assay). Proliferation assays and cytotoxic T cell assays will be performed on strong CD4+ and CD8+ responses respectively.

\subsection{Adverse Events}

See Appendix 1. 


\section{References}

1.Colditz GA, Brewer TF, Berkey CS, Wilson ME, Burdick E, Fineberg HV, Mosteller F. Efficacy of BCG vaccine in the prevention of TB. Meta-analysis of the published literature. $\mathrm{J}$ Am Med Assoc 1994;271:698-702

2.Stenger S, Modlin RL. T cell mediated immunity to Mycobacterium TB. Curr Op Micro 1999; 2: 89-93

3.Huygen K, Content J, Denis O, Montgomery DL, Yawman AM, Deck RR, DeWitt CM, Orme IM, Baldwin S, D’Souza C, Drowart A, Lozes E, Vandenbussche P, Van Vooren JP, Liu MA, Ulmer JB. Immunogenicity and protective efficacy of a TB DNA vaccine. Nature Medicine 1996;2:893-898

4.Tascon RE, Colston MJ, Ragno S, Stavropoulos E, Gregory D, Lowrie DB. Vaccination against tuberculosis by DNA injection. Nature Medicine 1996;2:888-892

5.Schneider J, Gilbert SC, Blanchard TJ, Hanke T, Robson KJ, Hannan CM, Becker M, Sinden

R, Smith GL, Hill AVS. Enhanced immunogenicity for CD8+ T cell induction and complete protective efficacy of malaria DNA vaccination by boosting with modified vaccinia virus Ankara. Nature Medicine 1998;4:397-402

6.Hanke T, Samuel RV, Blanchard TJ, Neumann VC, Allen TM, Boyson JE, Sharpe SA, Cook N, Smith GL, Watkins DI, Cranage MP, McMichael AJ. Effective induction of simian immunodeficiency virus-specific cytotoxic $\mathrm{T}$ lymphocytes in macaques by using a multiepitope gene and DNA prime-modified vaccinia virus Ankara boost vaccination regimen. J Virol 1999;73(9):7524-32

7.McShane H, Brookes R, Gilbert SC, Hill AVS. 2001. Enhanced immunogenicity of CD4+ T cell responses and protective efficacy of a DNA-MVA prime-boost vaccination regime in murine tuberculosis. Infection \& Immunity. 69:681-686

8.Paoletti E. Applications of pox virus vectors to vaccination: an update. Proc Natl Acad Sci USA 1996;93:11349-11353.

9.Smith GL, Cheng KC, Moss B. Vaccinia virus: an expression vector for genes from parasites. Parasitology 1986;92 Suppl:S109-17

10.Rodrigues M, Li S, Murata K, Rodriguez D, Rodriguez JR, Bacik I, Bennink JR, Yewdell JW, Garcia-Sastre A, Nussenzweig RS, et al. Influenza and vaccinia viruses expressing malaria CD8+ $\mathrm{T}$ and $\mathrm{B}$ cell epitopes. Comparison of their immunogenicity and capacity to induce protective immunity. J Immunol 1994 Nov 15;153(10):4636-48

11. Mackett M, Smith G, Moss B. Vaccinia virus: a selectable eukaryotic cloning and expression vector. Proc Natl Acad Sci USA 1982; 79: 7415-7419.

12.Panicali D, Paoletti E. Construction of poxviruses as cloning vectors: insertion of the thymidine kinase gene from herpes simplex virus into the DNA of infectious vaccinia virus. Proc Natl Acad Sci USA 1982; 79: 4927-4931.

13. Moss B. Genetically engineered poxviruses for recombinant gene expression, vaccination and safety. Proc Natl Acad Sci USA 1996; 93: 11341-11348.

14.Stickl H, Hochstein-Mintzel V, Mayr A, Huber HC, Schafer H, Holzner A. MVA vaccination against smallpox: clinical tests with an attenuated live vaccinia virus strain. Dtsch Med Wochenschr 1974 Nov 22;99(47):2386-92.

15. Mahnel H, Mayr A. Experiences with immunization against orthopox viruses of humans and animals using vaccine strain MVA. Berl Munch Tierarztl Wochenschr 1994 Aug;107(8):253-6. 
16.Mayr A, Stickl H, Muller HK, Danner K, Singer H. The smallpox vaccination strain MVA: marker, genetic structure, experience gained with the parenteral vaccination and behavior in organisms with a debilitated defence mechanism. Zentralbl Bakteriol [B] 1978 Dec;167(56):375-90.

17.Meyer H, Sutter G, Mayr A. Mapping of deletions in the genome of the highly attenuated vaccinia virus MVA and their influence on virulence. J Gen Virol 1991 May;72 ( Pt 5):1031-8. 18.Sutter G, Moss B. Nonreplicating vaccinia vector efficiently expresses recombinant genes. Proc Natl Acad Sci U S A 1992 Nov 15;89(22):10847-51.

19. Moss B. Genetically engineered poxviruses for recombinant gene expression, vaccination and safety. Proc Natl Acad Sci USA 1996; 93: 11341-11348.

20. Sutter G, Moss B. Novel vaccinia vector derived from the host range restricted and highly attenuated MVA strain of vaccinia virus. Dev Biol Stand 1995;84:195-200.

21.Sutter G, Wyatt LS, Foley PL, Bennink JR, Moss B. A recombinant vector derived from the host range-restricted and highly attenuated MVA strain of vaccinia virus stimulates protective immunity in mice to influenza virus. Vaccine 1994 Aug;12(11):1032-40.

22.Hirsch VM, Fuerst TR, Sutter G, Carroll MW, Yang LC, Goldstein S, Piatak M Jr, Elkins WR, Alvord WG, Montefiori DC, Moss B, Lifson JD. Patterns of viral replication correlate with outcome in simian immunodeficiency virus (SIV)-infected macaques: effect of prior immunization with a trivalent SIV vaccine in modified vaccinia virus Ankara. J Virol 1996 Jun;70(6):3741-52.

23.Wyatt LS, Shors ST, Murphy BR, Moss B. Development of a replication-deficient recombinant vaccinia virus vaccine effective against parainfluenza virus 3 infection in an animal model. Vaccine 1996 Oct;14(15):1451-8.

24.Carroll MW, Overwijk WW, Chamberlain RS, Rosenberg SA, Moss B, Restifo NP. Highly attenuated modified vaccinia virus Ankara (MVA) as an effective recombinant vector: a murine tumor model. Vaccine 1997 Mar;15(4):387-94.

25. Horwitz MA, Lee BW, Dillon BJ, Harth G. Protective immunity against tuberculosis induced by vaccination with major extracellular proteins of Mycobacterium tuberculosis.

Proc.Natl.Acad.Sci.U.S.A. 92, 1530-1534.

26. Belisle JT et al. Role of the major antigen of mycobacterium TB in cell wall biogenesis. Science 1997;276:1420-1422

27. WHO (EPI). Immunisation schedules in the WHO European Region, 1995. WHO Wkly Epidem Rec 1995; 70 (31):221-227 


\section{APPENDIX 1}

\section{ADVERSE EVENTS}

\section{Definition and Grading Intensity of Adverse Events}

An adverse event is defined as any unintended change in the body structure (signs) or body function (symptoms), whether or not considered related to test product. During the entire study, subjects will be instructed to report all adverse events. All adverse events, whether volunteered, elicited or noted on physical examination, will be recorded throughout the study.

The severity of adverse events will be categorized as follows:

- $\quad$ MILD = Experience that is minor and does not cause significant discomfort to subject or change in activities of daily living (ADLs); subject is aware of symptoms but symptoms are easily tolerated.

- $\quad$ MODERATE = Experience is an inconvenience or concern to the subject and causes interference with ADLs but the subject is able to continue with ADLs.

- SEVERE = Experience significantly interferes with ADLs and the subject is incapacitated and/or unable to continue with ADLs.

\section{Criteria for Determining Relationship to Test Product}

The Investigator will make a determination of the relationship of the adverse event to the test product. The relationship to test product of all adverse events will be classified according to the following guidelines:

- $\quad$ NOT RELATED = Data available to clearly identify an alternative cause of the reaction, e.g., hemorrhage due to mechanical injury.

- UNLIKELY

$\bullet \quad$ Reasonable temporal relation to vaccination, BUT

$\bullet \quad$ Unlabeled/unexpected reaction, AND

•• The reaction can be reasonably explained by other factors (such as interventions), AND

•• $\quad$ Negative de-challenge, if available, OR

$\bullet \quad$ No reasonable temporal relation to vaccination.

\section{- $\quad$ POSSIBLE}


•• Reasonable temporal relation to vaccination, AND

- Labeled/expected reaction, OR

$\bullet \quad$ Unlabeled/unexpected reaction, BUT

- Other factors could have caused or contributed to the reaction (such as subject's clinical state, concomitant therapy, and/or other interventions).

- $\quad$ PROBABLE

$\bullet \quad$ Reasonable temporal relation to vaccination, AND

$\bullet \quad$ Labeled/expected reaction, AND

$\bullet \quad$ The reaction cannot be reasonably explained by other factors (such as the subject's clinical state, concomitant therapy, and/or other interventions).

- $\quad$ HIGHLY PROBABLE

$\bullet \quad$ Reasonable temporal relation to vaccination, AND

-• Labeled/expected reaction, AND

- $\quad$ The reaction cannot be reasonably explained by other factors (such as the subject's clinical state, concomitant therapy, and/or other interventions), AND

$\bullet \quad$ Positive de-challenge, if applicable, AND

$\bullet \quad$ Positive re-challenge, OR

$\bullet \quad$ Application/vaccination site reaction.

The following adverse events are considered "serious reportable adverse events:”

- $\quad$ Death of a subject or life threatening events.

- Hospitalization (other than elective procedures or outpatient observation of $<24$ hour duration) or prolongation of hospitalization.

- $\quad$ Cancer or congenital anomaly.

- $\quad$ Chronic or permanent disability.

- $\quad$ Overdose.

- $\quad$ Any serious adverse event (i.e., an adverse event that is graded as serious or life-threatening in appendix 2). 


\section{APPENDIX 2}

\section{Table for Grading Severity of Adult Adverse Experiences for Vaccine Trials}

\section{Guidelines}

ABBREVIATIONS: Abbreviations utilized in this Table include:

$\begin{array}{llll}\text { ULN } & =\text { Upper Limit of Normal } & \text { LLN } & =\text { Lower Limit of Normal } \\ \mathrm{R}_{\mathrm{x}} & =\text { Therapy } & \text { Req } & =\text { Required } \\ \text { Mod } & =\text { Moderate } & \text { IV } & =\text { Intravenous } \\ \text { ADL } & =\text { Activities of Daily Living } & \text { Dec } & =\text { Decreased }\end{array}$

ESTIMATING SEVERITY GRADE

For abnormalities NOT found elsewhere in the Toxicity Table use the scale below to estimate grade of severity:

GRADE $1 \quad$ Mild Transient or mild discomfort (<48 hours); no medical intervention/therapy required.

GRADE 2 Moderate Mild to moderate limitation in activity - some assistance may be needed; no or minimal medical intervention/therapy required.

GRADE $3 \quad$ Severe Marked limitation in activity, some assistance usually required; medical intervention/therapy required, hospitalization possible.

GRADE 4 Life- threatening Extreme limitation in activity, significant assistance required; significant medical intervention/therapy required, hospitalization or hospice care probable.

\section{SERIOUS OR LIFE-THREATENING Adverse Events}

ANY clinical event deemed by the clinician to be serious or life-threatening should be considered a Grade 4 Adverse Event. Clinical events considered to be serious or life-threatening include, but are not limited to: seizures, coma, tetany, diabetic ketoacidosis, disseminated intravascular coagulation, diffuse petechiae, paralysis, acute psychosis, severe depression.

\section{MISCELLANEOUS}

- When two values are used to define the criteria for each parameter, the lowest values will appear first.

- $\quad$ Parameters are generally grouped by body system.

- $\quad$ Some protocols may have additional protocol specific grading criteria. 


\section{APPENDIX 2 continued - Modified Table for Grading Severity of Adult Adverse Experiences for Vaccine Trials}

\begin{tabular}{|c|c|c|c|c|}
\hline Parameter & $\begin{array}{l}\text { Grade } 1 \\
\text { Mild }\end{array}$ & $\begin{array}{c}\text { Grade } 2 \\
\text { Moderate }\end{array}$ & $\begin{array}{l}\text { Grade } 3 \\
\text { Severe }\end{array}$ & $\begin{array}{c}\text { Grade } 4 \\
\text { Potentially } \\
\text { Life-Threatening }\end{array}$ \\
\hline $\begin{array}{l}\text { POTASSIUM } \\
\text { Hyperkalemia } \\
\text { Hypokalemia } \\
\end{array}$ & $\begin{array}{l}5.0-5.5 \mathrm{meq} / \mathrm{L} \\
3.2-3.4 \mathrm{meq} / \mathrm{L} \\
\end{array}$ & $\begin{array}{l}5.6-6.0 \mathrm{meq} / \mathrm{L} \\
2.9-3.1 \mathrm{meq} / \mathrm{L} \\
\end{array}$ & $\begin{array}{l}6.1-6.5 \mathrm{meq} / \mathrm{L} \\
2.5-2.8 \mathrm{meq} / \mathrm{L}\end{array}$ & $\begin{array}{l}>6.5 \mathrm{meq} / \mathrm{L} \\
<2.5 \mathrm{meq} / \mathrm{L}\end{array}$ \\
\hline $\begin{array}{l}\text { PHOSPHATE } \\
\text { Hypophosphatemia }\end{array}$ & $2.0-2.4 \mathrm{mg} / \mathrm{dL}$ & $1.5-1.9 \mathrm{mg} / \mathrm{dL}$ & $1.0-1.4 \mathrm{mg} / \mathrm{dL}$ & $<1.0 \mathrm{mg} / \mathrm{dL}$ \\
\hline $\begin{array}{l}\text { CALCIUM - (corrected } \\
\text { for albumin) } \\
\text { Hypocalcemia } \\
\text { Hypercalcemia }\end{array}$ & $\begin{array}{l}7.8-8.4 \mathrm{mg} / \mathrm{dL} \\
10.6-11.5 \mathrm{mg} / \mathrm{dL}\end{array}$ & $\begin{array}{l}7.0-7.7 \mathrm{mg} / \mathrm{dL} \\
11.6-12.5 \mathrm{mg} / \mathrm{dL}\end{array}$ & $\begin{array}{l}6.1-6.9 \mathrm{mg} / \mathrm{dL} \\
12.6-13.5 \mathrm{mg} / \mathrm{dL}\end{array}$ & $\begin{array}{l}<6.1 \mathrm{mg} / \mathrm{dL} \\
>13.5 \mathrm{mg} / \mathrm{dL}\end{array}$ \\
\hline $\begin{array}{l}\text { MAGNESIUM } \\
\text { Hypomagnesemia }\end{array}$ & $1.2-1.4 \mathrm{meq} / \mathrm{L}$ & $0.9-1.1 \mathrm{meq} / \mathrm{L}$ & $0.6-0.8 \mathrm{meq} / \mathrm{L}$ & $<0.6 \mathrm{meq} / \mathrm{L}$ \\
\hline $\begin{array}{l}\text { BILIRUBIN } \\
\text { Hyperbilirubinemia }\end{array}$ & $>1.0-1.5 \times$ ULN & $>1.5-2.5 \times$ ULN & $>2.5-5 \times$ ULN & $>5 \times$ ULN \\
\hline $\begin{array}{l}\text { GLUCOSE } \\
\text { Hypoglycemia } \\
\text { Hyperglycemia } \\
\text { (nonfasting and no prior } \\
\text { diabetes) } \\
\end{array}$ & $\begin{array}{l}55-84 \mathrm{mg} / \mathrm{dL} \\
118-160 \mathrm{mg} / \mathrm{dL}\end{array}$ & $\begin{array}{l}40-54 \mathrm{mg} / \mathrm{dL} \\
161-250 \mathrm{mg} / \mathrm{dL}\end{array}$ & $\begin{array}{l}30-39 \mathrm{mg} / \mathrm{dL} \\
251-500 \mathrm{mg} / \mathrm{dL}\end{array}$ & $\begin{array}{l}<30 \mathrm{mg} / \mathrm{dL} \\
>500 \mathrm{mg} / \mathrm{dL}\end{array}$ \\
\hline Triglycerides & ---- & $400-750 \mathrm{mg} / \mathrm{dL}$ & $751-1200 \mathrm{mg} / \mathrm{dL}$ & $>1200 \mathrm{mg} / \mathrm{dL}$ \\
\hline $\begin{array}{l}\text { URIC ACID } \\
\text { Hyperuricemia }\end{array}$ & $7.5-10.0 \mathrm{mg} / \mathrm{dL}$ & $10.1-12.0 \mathrm{mg} / \mathrm{dL}$ & $12.1-15.0 \mathrm{mg} / \mathrm{dL}$ & $>15.0 \mathrm{mg} / \mathrm{dL}$ \\
\hline $\begin{array}{l}\text { LIVER TRANS- } \\
\text { AMINASE (LFTs) } \\
\text { AST (SGOT) } \\
\text { ALT (SGPT) } \\
\text { GGT } \\
\text { Alk Phos } \\
\end{array}$ & $\begin{array}{l}1.25-2.5 \times \text { XLN } \\
1.25-3.0 \times \text { ULN } \\
1.25-2.5 \times \text { ULN } \\
1.25-2.5 \times \text { ULN }\end{array}$ & $\begin{array}{l}>2.5-5.0 \times \text { ULN } \\
>3.0-5.0 \times \text { ULN } \\
>2.5-5.0 \times \text { ULN } \\
>2.5-5.0 \times \text { ULN }\end{array}$ & $\begin{array}{l}>5.0-10.0 \times \mathrm{ULN} \\
>5.0-10.0 \times \mathrm{ULN} \\
>5.0-10.0 \times \mathrm{ULN} \\
>5.0-10.0 \times \mathrm{ULN}\end{array}$ & $\begin{array}{l}>10.0 \times \text { ULN } \\
>10.0 \times \text { ULN } \\
>10.0 \times \text { ULN } \\
>10.0 \times \text { ULN }\end{array}$ \\
\hline $\begin{array}{l}\text { PANCREATIC } \\
\text { ENZYMES } \\
\text { Amylase } \\
\text { Pancreatic amylase } \\
\text { Lipase }\end{array}$ & $\begin{array}{l}>1.0-1.5 \times \text { ULN } \\
>1.0-1.5 \times \text { ULN } \\
>1.0-1.5 \times \text { ULN }\end{array}$ & $\begin{array}{l}>1.5-2.0 \times \text { ULN } \\
>1.5-2.0 \times \text { ULN } \\
>1.5-2.0 \times \text { ULN }\end{array}$ & $\begin{array}{l}>2.0-5.0 \times \text { ULN } \\
>2.0-5.0 \times \text { ULN } \\
>2.0-5.0 \times \text { ULN }\end{array}$ & $\begin{array}{l}>5.0 \times \text { ULN } \\
>5.0 \times \text { ULN } \\
>5.0 \times \text { ULN }\end{array}$ \\
\hline
\end{tabular}




\section{APPENDIX 2 continued - Modified Table for Grading Severity of Adult Adverse Experiences for Vaccine Trials}

\begin{tabular}{|c|c|c|c|c|}
\hline Parameter & $\begin{array}{l}\text { Grade } 1 \\
\text { Mild }\end{array}$ & $\begin{array}{l}\text { Grade } 2 \\
\text { Moderate }\end{array}$ & $\begin{array}{l}\text { Grade } 3 \\
\text { Severe }\end{array}$ & $\begin{array}{c}\text { Grade } 4 \\
\text { Potentially } \\
\text { Life-Threatening } \\
\end{array}$ \\
\hline \multicolumn{5}{|c|}{ CARDIOVASCULAR } \\
\hline $\begin{array}{l}\text { Cardiac } \\
\text { Arrhythmia }\end{array}$ & ------------ & $\begin{array}{c}\text { Asymptomatic; } \\
\text { transient dysrhythmia, } \\
\text { no } \mathrm{R}_{\mathrm{x}} \text { req }\end{array}$ & $\begin{array}{l}\text { Recurrent/persistent } \\
\text { dysrhythmia; } \\
\text { symptomatic } \mathrm{R}_{\mathrm{x}} \text { req }\end{array}$ & $\begin{array}{c}\text { Unstable } \\
\text { dysrhythmia, } \\
\text { hospitalization and } \\
\mathrm{R}_{\mathrm{x}} \text { req }\end{array}$ \\
\hline Hypertension & $\begin{array}{l}\text { Transient, increase }>20 \text { mm } \\
\text { Hg diastolic BP; no } \mathrm{R}_{x} \mathrm{req}\end{array}$ & $\begin{array}{c}\text { Recurrent; chronic } \\
\text { increase >20 mm Hg } \\
\text { diastolic BP; } \mathrm{R}_{\mathrm{x}} \text { req } \\
\end{array}$ & $\begin{array}{l}\text { Acute } \mathrm{R}_{\mathrm{x}} \text { req; outpatient } \mathrm{OR} \\
\text { hospitalization possible }\end{array}$ & $\begin{array}{c}\text { Hospitalization req } \\
\text { OR end organ } \\
\text { damage } \\
\end{array}$ \\
\hline Hypotension & $\begin{array}{c}\text { Transient orthostatic } \\
\text { hypotension with heart rate } \\
\text { increased by }>20 \text { beats } / \mathrm{min} \\
\text { OR decreased by }<10 \mathrm{~mm} \\
\text { Hg systolic BP, no } \mathrm{R}_{\mathrm{x}} \text { req }\end{array}$ & $\begin{array}{r}\text { Symptoms OR BP } \\
\text { decreased by }<20 \mathrm{~mm} \\
\text { Hg systolic, correctable } \\
\text { with oral fluid } \mathrm{R}_{\mathrm{x}}\end{array}$ & $\begin{array}{l}\text { IV fluid req OR } \\
\text { hospitalization }\end{array}$ & $\begin{array}{c}\text { Mean arterial } \\
\text { pressure }<60 \mathrm{~mm} \\
\text { Hg, OR end organ } \\
\text { damage, OR } \\
\text { shock, vasopressor } \\
\mathrm{R}_{\mathrm{x}} \text { req } \\
\end{array}$ \\
\hline Pericarditis & Minimal effuision & $\begin{array}{c}\text { Mild/mod } \\
\text { asymptomatic effusion, } \\
\text { no } \mathrm{R}_{\mathrm{x}} \\
\end{array}$ & $\begin{array}{l}\text { Symptomatic effusion, pain, } \\
\text { EKG changes }\end{array}$ & $\begin{array}{c}\text { Tamponade OR } \\
\text { pericardiocentesis } \\
\text { OR surgery req } \\
\end{array}$ \\
\hline $\begin{array}{l}\text { Hemorrhage, } \\
\text { blood loss }\end{array}$ & ------------ & $\begin{array}{l}\text { Mildly symptomatic, no } \\
\mathrm{R}_{\mathrm{x}} \text { req }\end{array}$ & $\begin{array}{l}\text { Gross blood loss OR 1-2 } \\
\text { units transfused }\end{array}$ & $\begin{array}{l}\text { Massive blood } \\
\text { loss OR }>2 \text { units } \\
\text { transfused }\end{array}$ \\
\hline \multicolumn{5}{|c|}{ GASTROINTESTINAL } \\
\hline Nausea & $\begin{array}{c}\text { Mild OR transient; } \\
\text { reasonable intake maintained }\end{array}$ & $\begin{array}{c}\text { Mod discomfort OR } \\
\text { intake decreased for }<3 \\
\text { days }\end{array}$ & $\begin{array}{c}\text { Severe discomfort OR } \\
\text { minimal intake for } \geq 3 \text { days }\end{array}$ & Hospitalization req \\
\hline Vomiting & $\begin{array}{c}\text { Mild OR transient; } 2-3 \\
\text { episodes per day OR mild } \\
\text { vomiting lasting }<1 \text { week }\end{array}$ & $\begin{array}{l}\text { Mod OR persistent; 4-5 } \\
\text { episodes per day; OR } \\
\text { vomiting lasting } \geq \text { week }\end{array}$ & $\begin{array}{c}\text { Severe vomiting of all } \\
\text { food/fluids in } 24 \text { hrs OR } \\
\text { orthostatic hypotension OR } \\
\text { IV } R_{x} \text { req }\end{array}$ & $\begin{array}{l}\text { Hypotensive shock } \\
\text { OR hospitalization } \\
\text { req for } I V R_{x} \text { req }\end{array}$ \\
\hline Diarrhea & $\begin{array}{l}\text { Mild OR transient; 3-4 loose } \\
\text { stools per day OR mild } \\
\text { diarrhea lasting }<1 \text { week }\end{array}$ & $\begin{array}{l}\text { Mod OR persistent; 5- } \\
10 \text { loose stools per day } \\
\text { OR diarrhea lasting } \geq 1 \\
\text { week }\end{array}$ & $\begin{array}{c}\text { >10 loose stools/day bloody } \\
\text { diarrhea; OR orthostatic } \\
\text { hypotension OR electrolyte } \\
\text { imbalance, }>2 \text { L IV fluid req }\end{array}$ & $\begin{array}{l}\text { Hypotensive shock } \\
\text { OR severe } \\
\text { electrolyte } \\
\text { imbalance }\end{array}$ \\
\hline $\begin{array}{l}\text { Oral Discomfort/ } \\
\text { Dysphagia }\end{array}$ & $\begin{array}{l}\text { Mild discomfort, no } \\
\text { difficulty swallowing }\end{array}$ & $\begin{array}{c}\text { Difficulty swallowing } \\
\text { but able to eat and drink }\end{array}$ & Unable to swallow solids & $\begin{array}{l}\text { Unable to drink } \\
\text { fluids; IV fluids } \\
\text { req }\end{array}$ \\
\hline Constipation & & $\begin{array}{l}\text { Moderate abdominal } \\
\text { pain } 78 \text { hours with } \\
\text { impaction require } \\
\text { outpatient prescription }\end{array}$ & $\begin{array}{l}\text { Requiring disimpaction or } \\
\text { hospital treatment }\end{array}$ & $\begin{array}{c}\text { Distention with } \\
\text { vomiting OR } \\
\text { obstipation }\end{array}$ \\
\hline
\end{tabular}




\section{APPENDIX 2 - Modified Table for Grading Severity of Adult Adverse Experiences for Vaccine Trials}

\begin{tabular}{|c|c|c|c|c|}
\hline Parameter & $\begin{array}{l}\text { Grade } 1 \\
\text { Mild }\end{array}$ & $\begin{array}{c}\text { Grade } 2 \\
\text { Moderate }\end{array}$ & $\begin{array}{c}\text { Grade } 3 \\
\text { Severe }\end{array}$ & $\begin{array}{c}\text { Grade } 4 \\
\text { Potentially Life- } \\
\text { Threatening }\end{array}$ \\
\hline $\begin{array}{l}\text { Cough (for aerosol } \\
\text { studies) }\end{array}$ & Transient; no $\mathrm{R}_{\mathrm{x}}$ & $\begin{array}{l}\text { Treatment associated } \\
\text { cough; inhaled } \\
\text { bronchodilator }\end{array}$ & $\begin{array}{l}\text { Uncontrolled cough; } \\
\text { systemic } \mathrm{R}_{\mathrm{x}} \text { req }\end{array}$ & -------------- \\
\hline Bronchospasm Acute & $\begin{array}{c}\text { Transient; no } \mathrm{R}_{\mathrm{x}} \text {; } \\
\text { FEV1 or peak flow } \\
\text { reduced to } 70 \%-80 \%\end{array}$ & $\begin{array}{l}\mathrm{R}_{\mathrm{x}} \text { req; normalizes with } \\
\text { bronchodilator; FEV1 or } \\
\text { peak flow } 50 \% \text { - } 69 \%\end{array}$ & $\begin{array}{l}\text { No normalization with } \\
\text { bronchodilator; FEV1 or } \\
\text { peak flow } 25 \% \text { - } 49 \% \text {, } \\
\text { retractions }\end{array}$ & $\begin{array}{c}\text { Cyanosis; FEV1 or } \\
\text { peak flow }<25 \% \\
\text { OR intubated }\end{array}$ \\
\hline Dyspnea & Dyspnea on exertion & $\begin{array}{c}\text { Dyspnea with normal } \\
\text { activity }\end{array}$ & Dyspnea at rest & $\begin{array}{c}\text { Dyspnea requiring } \\
\mathrm{O}_{2} \text { therapy }\end{array}$ \\
\hline \multicolumn{5}{|c|}{ NEUROLOGICAL } \\
\hline Neuro-cerebellar & $\begin{array}{l}\text { Slight incoordination } \\
\text { OR } \\
\text { Dysdiadochokinesia }\end{array}$ & $\begin{array}{c}\text { Intention tremor OR } \\
\text { dysmetria OR slurred } \\
\text { speech OR nystagmus }\end{array}$ & $\begin{array}{l}\text { Ataxia requiring assistance } \\
\text { to walk or arm } \\
\text { incoordination interfering } \\
\text { with ADLs }\end{array}$ & Unable to stand \\
\hline Neuro-psych/mood & & ---------------- & $\begin{array}{l}\text { Severe mood changes } \\
\text { requiring medical } \\
\text { intervention; suicidal } \\
\text { ideation }\end{array}$ & $\begin{array}{c}\text { Acute psychosis } \\
\text { req hospitalization } \\
\text {; suicidal } \\
\text { gesture/attempt }\end{array}$ \\
\hline $\begin{array}{l}\text { Parasthesia (burning, } \\
\text { tingling, etc.) }\end{array}$ & $\begin{array}{l}\text { Mild discomfort; no } \mathrm{R}_{\mathrm{x}} \\
\text { req }\end{array}$ & $\begin{array}{l}\text { Mod discomfort; non- } \\
\text { narcotic analgesia required }\end{array}$ & $\begin{array}{c}\text { Severe discomfort; OR } \\
\text { narcotic analgesia req with } \\
\text { symptomatic improvement }\end{array}$ & $\begin{array}{c}\text { Incapacitating; OR } \\
\text { not responsive to } \\
\text { narcotic analgesia }\end{array}$ \\
\hline Neuro-motor & $\begin{array}{c}\text { Mild weakness in } \\
\text { muscle of feet but able } \\
\text { to walk and/or mild } \\
\text { increase or decrease in } \\
\text { reflexes }\end{array}$ & $\begin{array}{l}\text { Mod weakness in feet } \\
\text { (unable to walk on heels } \\
\text { and/or toes), mild } \\
\text { weakness in hands, still } \\
\text { able to do most hand tasks } \\
\text { and/or loss of previously } \\
\text { present reflex or } \\
\text { development of } \\
\text { hyperreflexia and/or } \\
\text { unable to do deep knee } \\
\text { bends to weakness }\end{array}$ & $\begin{array}{l}\text { Marked distal weakness } \\
\text { (unable to dorsiflex toes or } \\
\text { foot drop, and mod proximal } \\
\text { weakness e.g., in hands } \\
\text { interfering with ADLs } \\
\text { and/or requiring assistance } \\
\text { to walk and/or unable to rise } \\
\text { from chair unassisted }\end{array}$ & $\begin{array}{l}\text { Confined to bed or } \\
\text { wheel chair } \\
\text { because of muscle } \\
\text { weakness }\end{array}$ \\
\hline Neuro-sensory & $\begin{array}{l}\text { Mild impairment } \\
\text { (decreased sensation, } \\
\text { e.g., vibratory, } \\
\text { pinprick, hot/cold in } \\
\text { great toes) in focal } \\
\text { area or symmetrical } \\
\text { distribution }\end{array}$ & $\begin{array}{l}\text { Mod impairment (mod } \\
\text { decreased sensation, e.g., } \\
\text { vibratory, pinprick, } \\
\text { hot/cold to ankles) and/or } \\
\text { joint position or mild } \\
\text { impairment that is not } \\
\text { symmetrical }\end{array}$ & $\begin{array}{c}\text { Severe impairment } \\
\text { (decreased or loss of } \\
\text { sensation to knees or wrists) } \\
\text { or loss of sensation of at } \\
\text { least mod degree in multiple } \\
\text { different body sites (i.e., } \\
\text { upper and lower extremities) }\end{array}$ & $\begin{array}{c}\text { Sensory loss } \\
\text { involves limbs and } \\
\text { trunk }\end{array}$ \\
\hline
\end{tabular}


APPENDIX 2 continued - Modified Table for Grading Severity of Adult Adverse Experiences for Vaccine Trials

\begin{tabular}{|c|c|c|c|c|}
\hline Parameter & $\begin{array}{c}\text { Grade } 1 \\
\text { Mild }\end{array}$ & $\begin{array}{c}\text { Grade } 2 \\
\text { Moderate }\end{array}$ & $\begin{array}{l}\text { Grade } 3 \\
\text { Severe }\end{array}$ & $\begin{array}{c}\text { Grade } 4 \\
\text { Potentially } \\
\text { Life-Threatening }\end{array}$ \\
\hline Arthralgia/Arthritis & Arthralgia & $\begin{array}{l}\text { Arthralgia with joint } \\
\text { effusion or moderate } \\
\text { impairment of activity }\end{array}$ & $\begin{array}{l}\text { Frank arthritis with or } \\
\text { without effusion OR } \\
\text { resulting in severe } \\
\text { impairment of activity }\end{array}$ & --- \\
\hline Myalgia & $\begin{array}{c}\text { Myalgia without } \\
\text { limitation of activity }\end{array}$ & $\begin{array}{l}\text { Muscle tenderness at } \\
\text { other than injection site } \\
\text { or with moderate } \\
\text { impairment of activity }\end{array}$ & $\begin{array}{c}\text { Frank myonecrosis OR } \\
\text { with severe impairment } \\
\text { of activity }\end{array}$ & -------------- \\
\hline \multicolumn{5}{|c|}{ SKIN } \\
\hline "Skin (vaccination site) & \multicolumn{4}{|c|}{ Refer to Appendix 4 for evaluation of specific changes at site of vaccination } \\
\hline Skin (general) & $\begin{array}{l}\text { Scattered macular or } \\
\text { papular eruption or } \\
\text { erythema that is } \\
\text { asymptomatic }\end{array}$ & $\begin{array}{l}\text { Scattered macular or } \\
\text { papular eruption or } \\
\text { erythema with pruritus } \\
\text { or other associated } \\
\text { symptoms }\end{array}$ & $\begin{array}{c}\text { Generalized } \\
\text { symptomatic macular, } \\
\text { papular, or vesicular } \\
\text { eruption }\end{array}$ & $\begin{array}{l}\text { Exfoliative dermatitis } \\
\text { or ulcerating dermatitis }\end{array}$ \\
\hline \multicolumn{5}{|c|}{ URINALYSIS } \\
\hline $\begin{array}{l}\text { Proteinuria: } \\
\text { Random urine }\end{array}$ & $1+$ & $2-3+$ & $4+$ & Nephrotic syndrome \\
\hline $\begin{array}{l}\text { Proteinuria: } \\
24 \text { hour urine }\end{array}$ & $\begin{array}{l}200 \mathrm{mg}-1 \mathrm{~g} \text { loss/day } \\
\text { OR }<0.3 \% \text { OR }<3 \text { g/l }\end{array}$ & $\begin{array}{c}1-2 \text { g loss/day OR } \\
0.3-1.0 \% \text { OR } 3-10 \\
\text { g/l }\end{array}$ & $\begin{array}{c}2-3.5 \mathrm{~g} \text { loss/day OR } \\
>1.0 \% \text { OR }>10 \mathrm{~g} / \mathrm{l}\end{array}$ & $\begin{array}{l}\text { Nephrotic syndrome } \\
\text { OR >3.5 g loss/day }\end{array}$ \\
\hline $\begin{array}{r}\text { Proteinuria: } \\
\text { Hematuria }\end{array}$ & $\begin{array}{c}\text { Microscopic only } \leq 10 \\
\text { RBC/HPF }\end{array}$ & $>10 \mathrm{RBC} / \mathrm{HPF}$ & $\begin{array}{l}\text { Gross, with or without } \\
\text { clots OR RBC casts }\end{array}$ & $\begin{array}{l}\text { Obstructive OR } \\
\text { transfusion req }\end{array}$ \\
\hline
\end{tabular}




\section{APPENDIX 2 continued - Modified Table for Grading Severity of Adult Adverse Experiences for Vaccine Trials}

\begin{tabular}{|c|c|c|c|c|}
\hline Parameter & $\begin{array}{l}\text { Grade } 1 \\
\text { Mild }\end{array}$ & $\begin{array}{c}\text { Grade } 2 \\
\text { Moderate }\end{array}$ & $\begin{array}{l}\text { Grade } 3 \\
\text { Severe }\end{array}$ & $\begin{array}{c}\text { Grade } 4 \\
\text { Potentially } \\
\text { Life-Threatening }\end{array}$ \\
\hline \multicolumn{5}{|c|}{ MISCELLANEOUS } \\
\hline $\begin{array}{l}\text { Fever } \\
\text { Oral }>12 \text { hours }\end{array}$ & $\begin{array}{c}37.7-38.9^{\circ} \mathrm{C} \\
\left(100.0-101.5^{\circ} \mathrm{F}\right)\end{array}$ & $\begin{array}{c}39.0-39.5^{\circ} \mathrm{C} \\
\left(101.6-102.9^{\circ} \mathrm{F}\right) \\
\text { OR max temp of } \\
103^{\circ} \mathrm{F}\end{array}$ & $\begin{array}{c}39.8-40.5^{\circ} \mathrm{C} \\
\left(103-105^{\circ} \mathrm{F}\right) \\
\text { OR max temp of } \\
103.5^{\circ} \mathrm{F}\end{array}$ & $\begin{array}{c}>40.5^{\circ} \mathrm{C}\left(105^{\circ} \mathrm{F}\right) \\
\text { OR max temp of }>105^{\circ} \mathrm{F}\end{array}$ \\
\hline Headache & $\begin{array}{l}\text { Mild, no } \mathrm{R}_{\mathrm{x}} \text { req, OR non- } \\
\text { narcotic analgesia } \mathrm{R}_{\mathrm{x}}\end{array}$ & $\begin{array}{l}\text { Mod; OR responds to } \\
\text { initial narcotic } R_{x}\end{array}$ & $\begin{array}{c}\text { Severe; intractable; OR } \\
\text { requiring repeated } \\
\text { narcotic } \mathrm{R}_{\mathrm{x}}\end{array}$ & $\begin{array}{l}\text { Requiring hospitalization, } \\
\text { associated with neurologic, } \\
\text { respiratory or } \\
\text { cardiovascular } \\
\text { abnormalities }\end{array}$ \\
\hline Allergic Reaction & $\begin{array}{c}\text { Pruritus without rash at } \\
\text { injection site }\end{array}$ & $\begin{array}{l}\text { Localized urticaria at } \\
\text { injection site }\end{array}$ & $\begin{array}{l}\text { Generalized urticaria } \\
\text { angioedema }\end{array}$ & Anaphylaxis \\
\hline 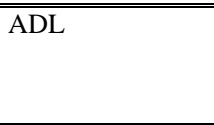 & $\begin{array}{c}\text { Normal activity reduced } \\
<48 \text { hours }\end{array}$ & $\begin{array}{l}\text { Normal activity } \\
\text { reduced } 25-50 \%>48 \\
\text { hours }\end{array}$ & $\begin{array}{c}\text { Normal activity reduced } \\
>50 \% \text {; cannot work }>48 \\
\text { hours } \\
\end{array}$ & "Unable to care for self \\
\hline Eye & & $\begin{array}{c}\text { Mild pain, visual } \\
\text { changes, conjunctival } \\
\text { erythema, abnormal } \\
\text { slit lamp }\end{array}$ & $\begin{array}{l}\text { Loss of vision, clinically } \\
\text { diagnosed uveitis, mod- } \\
\text { severe pain, glaucoma }\end{array}$ & --- \\
\hline
\end{tabular}

